\title{
Ataxie de Friedreich et mitochondrie : le puzzle reconstitué
}

L'ataxie de Friedreich est une maladie neurodégénérative autosomique récessive touchant environ une personne sur 50000 . Le gène responsable de la maladie a été identifié par clonage positionnel par l'équipe de Michel Koenig en 1996 et code pour une petite protéine de fonction inconnue de 210 acides aminés, la frataxine $[1,2]$. Cette ataxie est la première maladie génétique autosomique récessive dans laquelle a été observée une expansion de triplets. Effectivement, la quasi-totalité des allèles mutés correspondent à une expansion d'une répétition GAA variant de 150 à 1000 triplets chez les malades contre 7 à 25 dans la population normale. Cette expansion est située dans le premier intron du gène de la frataxine et entraîne une diminution quantitative du messager. On est donc en présence d'une maladie par perte de fonction de la frataxine.

La séquence protéique de la frataxine n'a donné aucune indication quant à sa fonction, mais des analogues de la frataxine ont été identifiés dans d'autres espèces grâce aux banques de données, dont un chez la levure Saccharomyces cerevisiae $(\mathrm{m} / \mathrm{s}$ $n^{\circ} 10$, vol. 13, p. 1216) [1-3]. Le gène correspondant, YFH1, avait été préalablement identifié au cours du séquençage systématique du génome de levure et, également, par complémentation d'un mutant de levure incapable de pousser sur un milieu pauvre en fer. L'analogie de séquence protéique entre la frataxine et le produit du gène $Y F H 1$ porte essentiellement sur les 100 derniers acides aminés de la frataxine. La partie amino-terminale de l'analogue de les caractéristiques d'une préséquence de ciblage mitochondrial. Cela a évidemment conduit à proposer que la frataxine serait une protéine mitochondriale et effectivement des études d'expression de la frataxine fusionnée à la $\beta$-galactosidase ont montré une localisation mitochondriale de la frataxine dans les cellules HeLa [4]. Pour comprendre le rôle de la frataxine et de son analogue Yfh1p chez la levure, le gène YFH1 a été délété et la souche résultante s'est révélée être une souche $r h o^{-}$, c'est-à-dire présentant un déficit de la chaîne respiratoire mitochondriale. De plus, les levures $\triangle Y F H 1$ accumulent des quantités impressionnantes de fer dans leurs mitochondries, jusqu'à environ dix fois la quantité observée dans les mitochondries d'une souche sauvage $[5,6]$. Par ailleurs, ces levures présentent une induction du système de transport à forte affinité pour le fer au niveau de la membrane plasmique. Le gène $Y F H 1$ coderait donc pour une protéine mitochondriale qui interviendrait dans le transport du fer à travers la membrane mitochondriale sans qu'il soit encore possible de savoir si cette protéine intervient dans l'entrée ou la sortie du fer. Mais qu'en est-il chez l'homme? Les caractéristiques cliniques de l'ataxie de Friedreich ont longtemps évoqué la possibilité d'une origine mitochondriale de la maladie. Effectivement, l'association d'atteintes d'organes différents (ataxie, cardiomyopathie, diabète) ainsi que le caractère évolutif de la maladie sont très évocateurs d'un déficit de la chaîne respiratoire mitochondriale [7]. Quelques travaux ont d'ailleurs rapporté l'existence de déficits de différentes enzymes mitochondriales sans que ces résultats puissent être confirmés par la suite. Il faut préciser que ces investigations étaient effectuées sur les lymphocytes ou le muscle de patients, tissus qui n'expriment pas la maladie. Or il est désormais bien établi que dans le cas des cytopathies mitochondriales il est indispensable d'explorer le tissu exprimant la maladie. Malheureusement, dans une atteinte essentiellement neurologique et cardiaque, l'accessibilité à de tels tissus pose bien évidemment des problèmes éthiques. On n'était donc pas près de sortir de ce cercle vicieux lorsqu'un malade atteint de cardiomyopathie, un peu trop vite caractérisée d'isolée, subit une biopsie endomyocardique pour étude de la chaîne respiratoire. Il faut rappeler que $40 \%$ à $50 \%$ des cardiomyopathies hypertrophiques idiopathiques sont dues à des déficits de la chaîne respiratoire [8]. Nous avons montré que cet enfant présentait un déficit de la chaîne respiratoire, mais d'un type particulièrement rare affectant les protéines fer-soufre de la chaîne respiratoire, au niveau des complexes I, II et III. Ce déficit des protéines fer-soufre incluait également l'aconitase, autre protéine fersoufre. Dans un deuxième temps, nous avons montré que ce déficit ne se retrouvait que dans le cœur, puisque le muscle, les lymphocytes et les fibroblastes de ce malade étaient parfaitement normaux. Dans ce deuxième temps, un autre élément particulièrement important est apparu, à savoir que ce malade présentait également une ataxie et un pied creux, signes majeurs de la maladie de Friedreich, et qui plus est une expansion de triplets GAA dans 
le premier intron du gène de la frataxine ! On avait donc enfin démontré que l'ataxie de Friedreich est une maladie mitochondriale due à un déficit des protéines fer-soufre. Cela a depuis été retrouvé chez deux autres patients [9]. Ce même déficit a aussi été identifié chez les levures $\triangle Y F H 1$.

Le lien avec les résultats observés chez la levure se fait alors tout naturellement. Il existe chez l'homme deux aconitases, une mitochondriale qui fait partie du cycle de Krebs et l'autre cytosolique qui acquiert une activité IRP (iron regulatory protein) quand la concentration en fer diminue dans la cellule. Dans cette situation, l'IRP se fixe à la partie 3' nontraduite des ARN messagers du récepteur de la transferrine, évitant ainsi leur dégradation et augmentant d'autant la capacité de la cellule d'importer du fer $\left(\mathrm{m} / \mathrm{s} n^{\circ} 10\right.$, vol. 9, p. 1145) [10]. La perte d'activité aconitase observée chez les patients atteints d'ataxie de Friedreich refléterait donc une diminution du fer cytosolique ayant pour conséquence de maintenir l'aconitase sous sa forme IRP. L'activation permanente du système d'import du fer dans la cellule conduirait, par homologie avec ce que l'on voit chez la levure, à son stockage dans les mitochondries. Cette surcharge de fer dans les mitochondries pourrait engendrer des radicaux oxygène hautement toxiques pour la chaîne respiratoire, tout particulièrement pour les protéines fer-soufre, d'où le déficit observé chez les patients.

Il reste donc maintenant à comprendre le rôle précis de la frataxine, à savoir comment elle intervient dans le transport du fer et avec quelles autres protéines elle interagit. Enfin, la compréhension des mécanismes physiopathologiques de la maladie ouvre maintenant le champ à la recherche de traitements permettant de réduire l'accumulation intramitochondriale de fer et de diminuer les conséquences du stress oxydatif qui en résulte.

A.R.

A.M.

P.R.

1. Campuzano V, Montermini L, Molto MD, et al. Friedreich's ataxia: autosomal recessive disease caused by an intronic GAA triplet repeat expansion. Science 1996; 271 : 1423-7.
2. Koenig M, Campuzano V, Cossée M, Mandel J. Ataxie de Friedreich: les expansions de triplets frappent encore. Med Sci 1996; 12: 431-5.

3. Gibson TJ, Koonin EV, Musco G, Pastore A, Bork P. Friedreich's ataxia protein: phylogenetic evidence for mitochondrial dysfunction. Trends Neurosci 1996 ; 19: 465-8.

4. Koutnitkova H, Foury F, Dollé P, Cazzalini O, Koenig M. Studies of mouse and yeast homologues indicate a mitochondrial function for the Friedreich's ataxia gene. Nat Genet 1997; 16 : 34551.

5. Foury $\mathrm{F}$ Cazzalini $\mathrm{O}$. Deletion of the yeast homologue of the human gene associated with Friedreich's ataxia elicits iron accumulation in mitochondria. FEBS Lett 1997; 411 : 373-7.

6. Babcock M, de Silva D, Oaks R, Davis-Kaplan S, Jiralerspong S, Montermini L, Pandolfo M, Kaplan J. Regulation of mitochondrial iron accumulation by Yfhlp, a putative homolog of frataxin. Science 1997; 276: 1709-12.

7. Rustin P, Munnich A, Rötig A. Dix ans d'exploration des maladies mitochondriales: une collaboration modèle entre chercheurs et cliniciens. Med Sci 1996; 12 (suppl Cnrs): 37-43.

8. Rustin P, Le Bidois J, Chretien D, Bourgeron T, Piéchaud JF, Rötig A, Munnich A, Sidi D. Endomyocardial biopsy for early detection of mitochondrial disorders in hypertrophic cardiomyopathies. J Pediatr 1994; 124: 224-8.

9. Rötig A, de Lonlay P, Chretien D, Foury F, Koenig M, Sidi D, Munnich A, Rustin P. Aconitase and mitochondrial iron-sulfur protein deficiency in Friedreich ataxia. Nat Genet 1997; 17. 215-7.

10. Kaptain S, Downey WE, Tang C, Philpott C, Haile D, Orloff DG, Harford JB, Rouault TA, Klausner RD. A regulated RNA binding protein also possesses aconitase activity. Proc Natl Acad Sci USA 1991; 88: 10109-13.
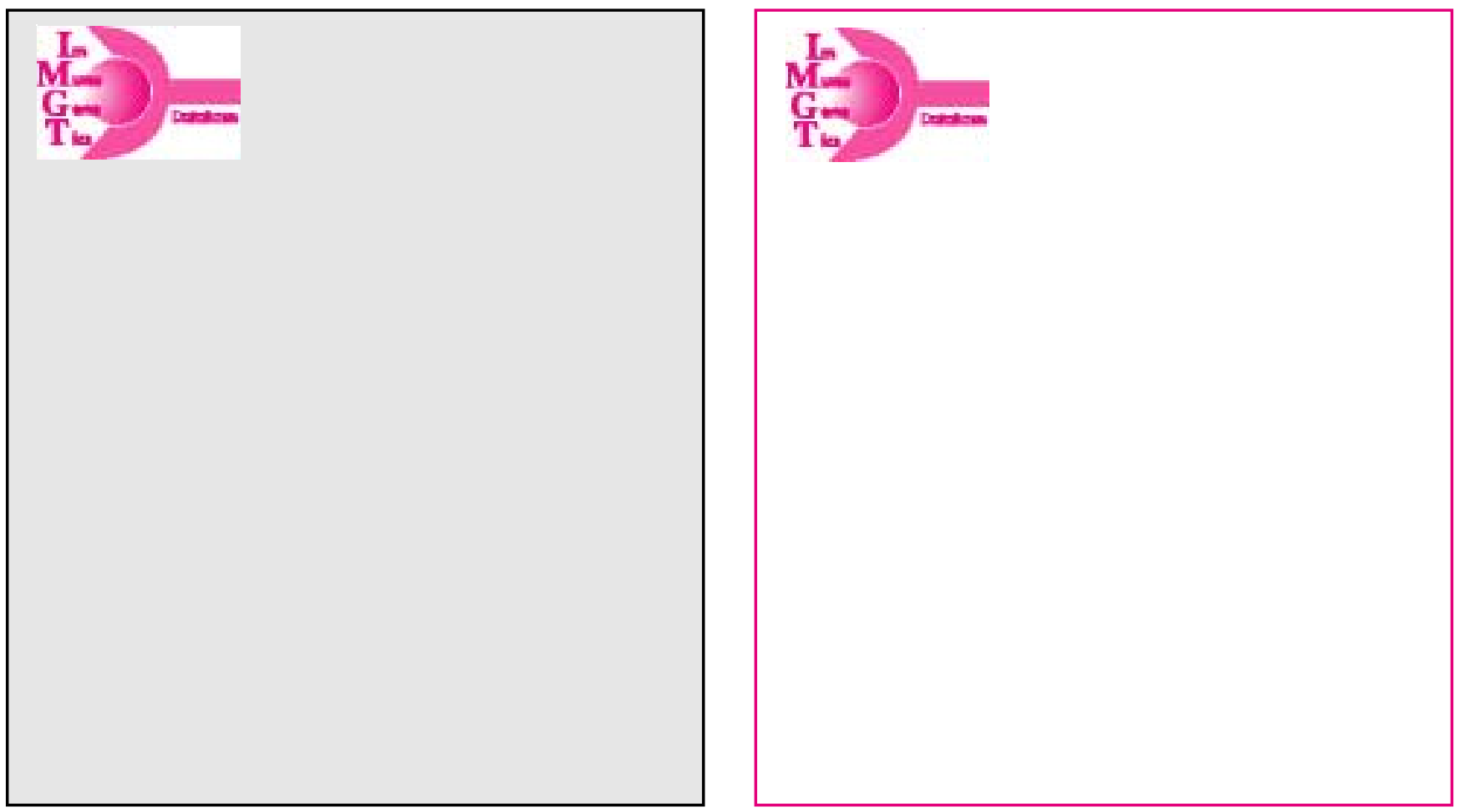